\title{
Higher risk of incomplete mesorectal excision and positive circumferential margin in low rectal cancer regardless of surgical technique
}

\author{
Alexander Ferko ${ }^{1}$, Julius Orhalmi ${ }^{1}$, Tomas Dusek ${ }^{1,2}$, Milan Chobola $^{1}$, Eva Hovorkova ${ }^{3}$, Dimitar Hadzi Nikolov ${ }^{3}$ \\ ${ }^{1}$ Department of Surgery, University Hospital Hradec Kralove, Charles University in Prague, Faculty of Medicine, Hradec Kralove, \\ Czech Republic \\ ${ }^{2}$ Department of Military Surgery, Faculty of Military Health Sciences, University of Defense, Hradec Kralove, Czech Republic \\ ${ }^{3}$ The Fingerland Department of Pathology, University Hospital Hradec Kralove, Charles University in Prague, Faculty of Medicine, \\ Hradec Kralove, Czech Republic
}

Videosurgery Miniinv 2014; 9 (4): 569-577

DOI: $10.5114 /$ wiitm.2014.45733

\begin{abstract}
Introduction: Currently, the predominant question is whether a laparoscopic approach is comparatively radical in comparison with an open access approach, especially in the circumferential resection margin and quality of the completeness of total mesorectal excision. These factors are important in determining the quality of surgical care as well as long-term results of the treatment.

Aim: This article focuses on the evaluation of circumferential resection margins and on the quality of mesorectal excision of middle and lower rectum tumors. In addition, laparoscopic and open techniques are compared.

Material and methods: Data were collected prospectively and stored in a rectal cancer registry over a 3-year period. The parameters studied were age, sex, body mass index, localization and topography of the tumor, clinical stage, neoadjuvant chemotherapy and its response, the type of surgery, character of the circumferential and distal margins, quality of the mesorectal excision, $P T$ and $p N$.

Results: One hundred and twenty-five patients were chosen for our study. Laparoscopy was performed in 53 operations and a conventional approach was performed in 72 operations. Complete mesorectal excision was achieved in $54.7 \%$ of laparoscopic operations versus $44.4 \%$ in the conventional technique; partially complete excision was performed in 20.8 and $12.5 \%$, respectively. Incomplete excisions were described in 24.5 and $43.1 \%(p=0.085)$. Positive circumferential margin occurred during laparoscopic surgery in 11 (20.8\%) patients, and in the case of conventional resection in $27(37.5 \%)$ patients $(p=0.044)$.

Conclusions: Our study showed comparable results between laparoscopic and open access procedures during rectal resection. The results achieved, in particular in the quality of the mesorectal excision and negative circumferential resection margin, show that the laparoscopic approach is comparable to conventional surgical techniques, with an adequate surgical outcome, in the treatment of rectal cancer.
\end{abstract}

Key words: colorectal cancer, total mesorectal excision, circumferential resection margin.

\section{Introduction}

The circumferential resection margin (CRM) and the quality of the completeness of total mesorectal excision (TME) are important factors. Without these, radical resections for rectal carcinoma cannot be evaluated $[1,2]$. It turns out that pathological cir-

\section{Address for correspondence}

Dr. Tomas Dusek, Department of Surgery, University Hospital Hradec Kralove, Charles University in Prague, Faculty of Medicine in Hradec Kralove, 581 Sokolska St, 50005 Hradec Kralove, Czech Republic, phone: +42 0608549389, e-mail: dusek.t@email.cz 
cumferential margin (pCRM) can be used as a direct indicator of oncological radicality, which significantly affects the outcome of treatment [1,3]. According to some, a positive pathological circumferential margin is a more significant independent predictive factor than the actual pT stage of the tumor [3].

The laparoscopic approach for resection of the rectum has been repeatedly analyzed for many years. Currently, the predominant question is whether a laparoscopic approach is comparatively radical in comparison with an open access approach, especially in the circumferential resection margin.

Our study is focused on the results of rectal resection of the aboral $10 \mathrm{~cm}$. One such study published on this subject was unfortunately hindered by methodological shortcomings. For example, patients with cancer of the upper rectum were included $[4,5]$, while amputation procedures for cancer of the distal third of the rectum were excluded [6]. In addition, overall patient selection was questionable $[7,8]$.

\section{Aim}

This article focuses on the evaluation of circumferential resection margins and on the quality of mesorectal excision of middle and lower rectum tumors. In addition, laparoscopic and open techniques are compared.

\section{Material and methods}

Our study includes patients with carcinoma of the middle and lower rectum who underwent surgery at the Department of Surgery at the University Hospital in Hradec Kralove in the period from January the $1^{\text {st }} 2010$ to December the 31 2012. Rectal cancer diagnosis was performed by standard procedures (colonoscopy, biopsy). Preoperative TNM staging was determined by abdominal computed tomography (CT). Almost all patients underwent a pretreatment pelvic nuclear magnetic resonance (NMR), and in some cases endorectal endosonography was also provided. The height of the lower edge of the tumor in all patients was based on clinical examination, including digital rectal or endorectal sonography and MRI measurement.

\section{Monitored data}

All observed data were prospectively entered into the registry for rectal cancer - ProMED. The factors investigated in the laparoscopic group and in the classic procedures were age, sex, body mass index, tumor location and its location in the rectal circumference, clinical stage, type of preoperative therapy and response to it, the type of surgery, tumor distance from the distal resection margins, quality of the total mesorectal excision, pathological tumor invasion and pathological evaluation of lymph nodes.

Patients in whom the lower tumor margin was higher than $10 \mathrm{~cm}$ from the anal verge were excluded. Compliance with this condition guaranteed the possibility of performing a total mesorectal excision. In addition, patients who have not undergone resection but only palliative derivative stoma were also excluded. Patients in whom the laparoscopic operations were converted were also excluded from the study. The last exclusion criterion was a complete pathological response (урT0) or if the tumor was not histologically found after non-radical endoscopic polypectomy. In these cases it was not possible to assess the circumferential margins.

The treatment strategy for each patient was determined by the decision of the oncosurgical multidisciplinary committee.

Preoperative oncological treatment was indicated in patients with locally advanced tumors ( $\mathrm{T} 2 \mathrm{~N}+$, $\mathrm{T} 3 \mathrm{~N}+, \mathrm{T} 4$ regardless of $\mathrm{N}+$ ). After the treatment was carried out, NMR re-staging took place and patients were operated on at least 8 weeks after completing the neoadjuvant cancer treatment.

Either a conventional or laparoscopic technique was employed for each operation. Abdominoperineal excision of the rectum and Hartmann's operation were also carried out. Since 2012, intersphincteric resection and extralevator abdominoperineal excision of the rectum has been performed at our institution. Adjuvant chemotherapy was indicated again on the basis of the conclusions of the oncosurgical committee, especially for patients with tumors of stage III and IV and high-risk patients in stage II.

\section{Histopathological evaluation of rectal resection}

A fresh specimen of the rectum was sent for histopathological examination immediately after terminating the resection phase of the operation. The histopathological evaluation was done by a knowledgeable pathologist in accordance with the "Histopathological Protocol for Rectal Cancer", which has been a standard part of our institutional evaluation 
for surgical treatment of rectal cancer since 2008 . The quality of the mesorectal excision was assessed by Quirke [1]. A positive circumferential resection margin is evaluated as a tumor or tumor-affected lymph nodes less than or equal to $1 \mathrm{~mm}$. A distance greater than $1 \mathrm{~mm}$ is considered negative.

\section{Statistical analysis}

For statistical evaluation the software NCSS 8 was used. For comparing the quantitative data we used either the two-sided $t$-test or the nonparametric Mann-Whitney, or the Kolmogorov-Smirnov test. To evaluate the qualitative data we used the $\chi^{2}$ test of independence in a contingency table or Fisher's exact test. Values of $p$ were obtained with the likelihood-ratio test and considered significance if $<0.05$.

\section{Results}

In the period from January the $1^{\text {st }} 2010$ to December $31^{\text {st }} 2012$ a total of 229 patients were operated on for rectal cancer at the Department of Surgery at the Faculty Hospital in Hradec Králové. Sixty-seven (29.3\%) patients with carcinoma of the upper rectum were excluded based on the given exclusion cri-

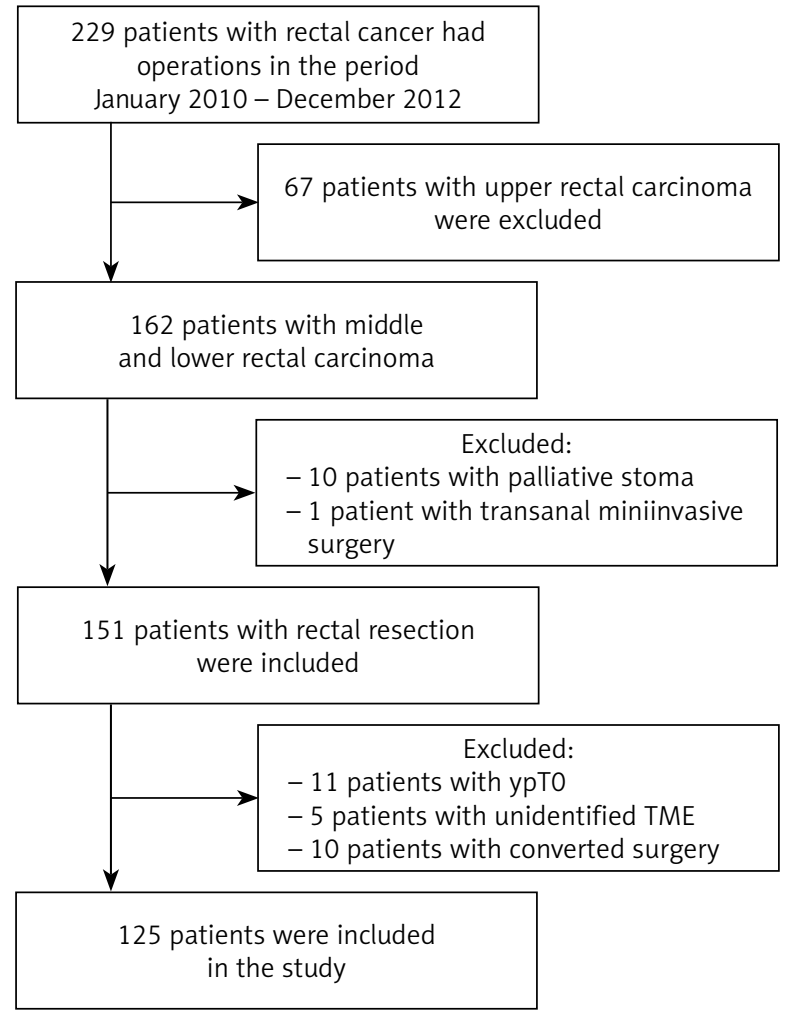

Figure 1. Study profile teria. From the remaining group of 162 patients with carcinoma of the middle and lower rectum, 11 further patients were excluded: 10 (6.2\%) who underwent only palliative stoma and 1 patient on whom transanal local surgery was performed. In addition, from the remaining 151 patients, 11 (7.3\%) patients with tumors of the middle and lower rectum were excluded (Figure 1). These patients had achieved a pathologically complete response following neoadjuvant chemoradiotherapy. Ten patients (6.2\%) were excluded due to converted surgery. The main reason was to achieve homogeneous groups. Five (3.3\%) other patients were not be intended quality of the mesorectal excision were also excluded. The total number of enrolled patients was 125: 76 men and 49 women, with an age range of 38-86 years.

Laparoscopy was performed in 53 cases, while a conventional approach was used in 72 cases (Figure 2). A diverting ileostomy was performed in 49 (36.3\%).

These two groups, the laparoscopic and conventional groups, differed significantly in body mass index (BMI, median $26.2 \mathrm{~kg} / \mathrm{m}^{2}$ vs. $\left.27.9 \mathrm{~kg} / \mathrm{m}^{2}\right)(p=$ $0.021)$. In both groups there was a higher number of men than women $(64.2 \%$ in the laparoscopic group and $58.3 \%$ in the conventional group). In the group of laparoscopic procedures, middle rectal carcinoma represented $52.8 \%$ of the procedures, tumors of the lower rectum $47.2 \%$. A conventional operation was

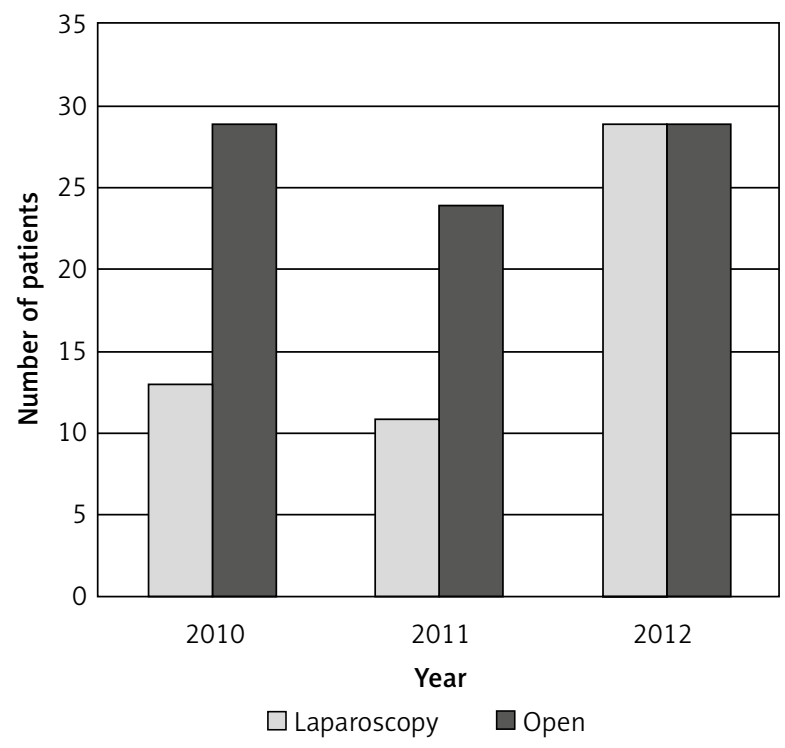

Figure 2. Low and mid third rectal cancer. Number of resection procedures in successive years 
Table I. Comparison of the laparoscopic and open group. Demographic and basic oncologic data

\begin{tabular}{|c|c|c|c|c|c|}
\hline \multirow[t]{2}{*}{ Parameter } & \multicolumn{2}{|c|}{ Laparoscopic } & \multicolumn{2}{|c|}{ Open } & \multirow[t]{2}{*}{ Value of $p$} \\
\hline & $n$ & $\%$ & $n$ & $\%$ & \\
\hline Number of patients & 53 & & 72 & & \\
\hline Age [years]: & & & & & 0.63 \\
\hline Mean & 65.4 & & 66.4 & & \\
\hline Median & 65 & & 66 & & \\
\hline Range & $40-85$ & & $38-86$ & & \\
\hline Gender: & & & & & 0.51 \\
\hline Male & 34 & 64.2 & 42 & 58.3 & \\
\hline Female & 19 & 35.8 & 30 & 41.7 & \\
\hline BMI [kg/m²]: & & & & & 0.021 \\
\hline Mean & 26.6 & & 28.4 & & \\
\hline Median & 26.2 & & 27.9 & & \\
\hline Range & $19.4-38.2$ & & $19.9-41.9$ & & \\
\hline Tumor location: & & & & & 0.54 \\
\hline Middle rectum $(5-10 \mathrm{~cm})$ & 28 & 52.8 & 34 & 47.2 & \\
\hline Lower rectum $(0-5 \mathrm{~cm})$ & 25 & 47.2 & 38 & 52.8 & \\
\hline Tumor topography: & & & & & 0.19 \\
\hline Circular & 11 & 20.8 & 24 & 34.2 & \\
\hline Anterior & 10 & 18.9 & 19 & 27.1 & \\
\hline Dorsal & 13 & 24.5 & 9 & 12.9 & \\
\hline Lateral on the right & 9 & 17 & 9 & 12.9 & \\
\hline Lateral on the left & 10 & 18.9 & 9 & 12.9 & \\
\hline Clinical stage: & & & & & 0.17 \\
\hline I & 23 & 43.4 & 21 & 29.2 & \\
\hline$\|$ & 8 & 15.1 & 21 & 29.2 & \\
\hline III & 15 & 28.3 & 17 & 23.6 & \\
\hline IV & 7 & 13.2 & 13 & 18.1 & \\
\hline
\end{tabular}

performed for middle rectal tumors in $47.2 \%$ of the cases and lower rectum in $52.8 \%$. In the group of middle rectal tumors there were 14 patients $(22.2 \%)$ with tumor localization within $9-10 \mathrm{~cm}$ from the anal verge.

Distribution based on clinical stage and type of surgery was not statistically significant (stage I $43.4 \%$. vs. $29.2 \%$, stage II $-15.1 \%$ vs. $29.2 \%$, stage III $-28.3 \%$ vs. $23.6 \%$ and stage IV $-13.2 \%$ vs. $18.1 \%)$ (Table I).
Ninety-seven (77.6\%) patients underwent total preoperative oncologic treatment, while $60.4 \%$ of the patients underwent chemoradiotherapy before the laparoscopic procedure, and $52.8 \%$ before conventional resection. Eight patients (2 vs. 6) were irradiated in long mode, and 19 patients (5 vs. 14) in short mode. Twenty-eight patients received no preoperative oncological treatment. No statistically significant difference was found in response to neoadjuvant therapy ( $p=$ 0.098), where a partial pathological response in the 
laparoscopic group occurred in $45.3 \%$ of the patients, in contrast to $30.6 \%$ in the group with open surgery.

Laparoscopy was performed in 43 patients $(81.1 \%)$ by means of low anterior resection, including 4 intersphincteric resections. An abdominoperineal amputation was performed in 9 patients of this group (17\%), along with 1 (1.9\%) Hartmann's operation. In the group of open surgery 26 (36.1\%) low anterior resections were performed, 39 (54.2\%) abdominoperineal amputations and 7 (9.7\%) Hartmann's resections. A statistically significant difference ( $p=0.000001$ ) was observed between these groups. The difference was mainly due to the high number of low anterior resections in the laparoscopic group and more than $50 \%$ share of amputation procedures in the open surgery group (Table II).

\section{Histopathological aspects}

The first quality of TME, namely complete mesorectal excision, was achieved in $54.7 \%$ of patients in the laparoscopic group, compared with $44.4 \%$ in the group of patients operated on conventionally. Partially complete excision was performed in $20.8 \%$ and $12.5 \%$, respectively. Incomplete excision was described in $24.5 \%$ and $43.1 \%$, respectively. Differenc- es in mesorectal excision in both surgical approaches were not statistically significant.

A positive circumferential margin was described in the laparoscopic procedure in 11 (20.8\%) patients. In the case of an open resection, the number was 27 (37.5\%). The results between groups were statistically significant ( $p=0.044)$ (Table III).

The ypCRM (after neoadjuvant oncologic treatment) was evaluated and compared in selected group of patients treated preoperatively with the laparoscopic or conventional technique (Table IV). The results were not statistically significant (Fisher $p=0.11$ ). We suppose that a relatively small group of patients has been included in our study to demonstrate the difference between laparoscopic and conventional groups. The distance from the distal tumor resection margin was not statistically significant, but it was in favor of the open method (median $20.7 \mathrm{~mm}$ in laparoscopy, $24.2 \mathrm{~mm}$ in the conventional procedure) $(p=0.45)$.

\section{Discussion}

The benefits of laparoscopic resection of the rectum, which include reduced postoperative pain and

Table II. Comparison of the laparoscopic and open group. Factors connected with treatment

\begin{tabular}{|c|c|c|c|c|c|}
\hline \multirow[t]{2}{*}{ Parameter } & \multicolumn{2}{|c|}{ Laparoscopic } & \multicolumn{2}{|c|}{ Open } & \multirow[t]{2}{*}{ Value of $p$} \\
\hline & $n$ & $\%$ & $n$ & $\%$ & \\
\hline Neoadjuvant therapy: & & & & & 0.28 \\
\hline Without & 14 & 26.4 & 14 & 19.4 & \\
\hline Chemoradiotherapy & 32 & 60.4 & 38 & 52.8 & \\
\hline Radiotherapy - long course & 2 & 3.8 & 6 & 8.4 & \\
\hline Radiotherapy - short course & 5 & 9.4 & 14 & 19.4 & \\
\hline Response to therapy: & & & & & 0.098 \\
\hline Partial pathological response & 24 & 45.3 & 22 & 30.6 & \\
\hline Stable disease & 9 & 16.9 & 23 & 31.9 & \\
\hline Progressive disease & 3 & 5.7 & 4 & 5.6 & \\
\hline Not available & 17 & 32.1 & 23 & 31.9 & \\
\hline Type of procedure: & & & & & 0.000001 \\
\hline Low anterior resection & 43 & 81.1 & 26 & 36.1 & \\
\hline Abdominoperineal amputation & 9 & 17 & 39 & 54.2 & \\
\hline Hartmann's procedure & 1 & 1.9 & 7 & 9.7 & \\
\hline
\end{tabular}


Table III. Comparison of the laparoscopic and open group. Results of the pathological examination

\begin{tabular}{|c|c|c|c|c|c|}
\hline \multirow[t]{2}{*}{ Parameter } & \multicolumn{2}{|c|}{ Laparoscopic } & \multicolumn{2}{|c|}{ Open } & \multirow[t]{2}{*}{ Value of $p$} \\
\hline & $n$ & $\%$ & $n$ & $\%$ & \\
\hline \multicolumn{3}{|c|}{ Character of distal resection margin: } & & & 0.23 \\
\hline Positive & 3 & 5.7 & 10 & 13.9 & \\
\hline Negative & 50 & 94.3 & 62 & 86.1 & \\
\hline \multicolumn{3}{|c|}{ Distance from distal resection margin to tumor [mm]: } & & & 0.45 \\
\hline Mean & 20.7 & & 24.2 & & \\
\hline Median & 15 & & 20 & & \\
\hline Range & $0-60$ & & 0-99.9 & & \\
\hline \multicolumn{3}{|c|}{ Circumferential margin [mm]: } & & & 0.20 \\
\hline Mean & 7.2 & & 6.2 & & \\
\hline Median & 5 & & 2.8 & & \\
\hline Range & $0-40$ & & $0-45$ & & \\
\hline \multicolumn{3}{|c|}{ Character of circumferential margin: } & & & 0.044 \\
\hline$\leq 1 \mathrm{~mm}$ & 11 & 20.8 & 27 & 37.5 & \\
\hline$>1 \mathrm{~mm}$ & 42 & 79.2 & 45 & 62.5 & \\
\hline \multicolumn{3}{|c|}{ Total mesorectal excision quality: } & & & 0.085 \\
\hline Complete & 29 & 54.7 & 32 & 44.4 & \\
\hline Nearly complete & 11 & 20.8 & 9 & 12.5 & \\
\hline Incomplete & 13 & 24.5 & 31 & 43.1 & \\
\hline \multicolumn{6}{|l|}{ pT stage } \\
\hline \multicolumn{3}{|l|}{ Middle rectum: } & & & 0.074 \\
\hline pT1 & 2 & 7.1 & 1 & 2.9 & \\
\hline pT2 & 17 & 60.8 & 12 & 35.3 & \\
\hline pT3 & 9 & 32.1 & 19 & 55.9 & \\
\hline pT4 & 0 & 0 & 2 & 5.9 & \\
\hline \multicolumn{3}{|l|}{ Low rectum: } & & & 0.068 \\
\hline pT1 & 5 & 20.0 & 3 & 7.9 & \\
\hline pT2 & 10 & 40.0 & 9 & 23.7 & \\
\hline pT3 & 9 & 36.0 & 17 & 44.7 & \\
\hline pT4 & 1 & 4.0 & 9 & 23.7 & \\
\hline \multicolumn{3}{|c|}{ Number of harvested lymphatic nodes: } & & & 0.26 \\
\hline Mean & 17.2 & & 19.0 & & \\
\hline Median & 14 & & 16 & & \\
\hline Range & $2-55$ & & $6-55$ & & \\
\hline \multicolumn{3}{|l|}{ pN stage: } & & & 0.85 \\
\hline NO & 31 & 58.5 & 44 & 61.1 & \\
\hline $\mathrm{N}+$ & 22 & 41.5 & 28 & 38.9 & \\
\hline
\end{tabular}

faster recovery, with both early resumption of bowel function and shortened hospitalization, are very well known and confirmed by prospective clinical studies
[5, 7-11]. An important and strictly monitored parameter is the oncological aspects of laparoscopic procedures. When comparing the results of surgical 
Table IV. ypT results in laparoscopic and open group with regard to CRM

\begin{tabular}{|c|c|c|c|c|c|}
\hline \multirow[t]{2}{*}{ CRM } & \multicolumn{2}{|c|}{ Laparoscopic } & \multicolumn{2}{|c|}{ Open } & \multirow[t]{2}{*}{ Value of $p$} \\
\hline & $n$ & $\%$ & $n$ & $\%$ & \\
\hline Negative & 32 & 82.1 & 39 & 67.2 & 0.11 \\
\hline Positive & 7 & 17.9 & 19 & 32.8 & \\
\hline
\end{tabular}

treatment of rectal cancer, particular attention must be given to the varying anatomy and principles of surgical treatment, especially in tumors of the lower third. Unfortunately, many studies do not reflect the view of the divergent results of TME and CRM tumors above and below $10 \mathrm{~cm}$ from the anus. Incorporating upper rectum resections into procedures has led to an "improvement" in the results and thus distorted the results of many studies. It has been shown that while the prognosis of rectal cancer depends principally on the stage of disease at diagnosis, the incidence of local recurrence after resection of the rectum reflects the quality of the surgeon [8, 12-16].

Traction using laparoscopic instrumentation is difficult and can lead to tears in the mesorectum, which can also be the cause of the poor quality of the mesorectal excision. On the other hand, laparoscopy provides a detailed view of the pelvis and greater control over the management of the identification of the resection line, essential for the high quality of the oncologic outcome. Capnoperitoneum plays a role, which helps separate the avascular tissue layers [16, 17]. The open operation is facilitated by hand traction, which does not damage fat mesorectal tissue. It has been repeatedly demonstrated that total mesorectal excision is a significant milestone in reducing local recurrence [2].

Obese patients have larger mesorectal fat tissue, so handling and dissecting in tighter spaces makes identifying the avascular layer difficult. Identifying the layers also poses problems even in thin patients. The layer of perirectal fat is low, and dissection is closer to the intestinal wall and thus to the tumor, making it difficult to prevent tearing the mesorectum [4]. The BMI difference between these two groups was evaluated in our study and showed statistical significance, probably due to the fact that patients with a lower BMI were previously operated on laparoscopically. Some studies take into account the distribution of "internal" fat and pelvic proportions as factors to be considered in terms of surgical tactics [18]. Also sex of the patient plays an import- ant role. A woman and a man carry the risk of injury of the mesorectum and PCRM disruption. Injury of the mesorectum can be caused by a thin fat layer, especially in the ventral part of the mesorectum in a woman. However, the combination of a narrow small pelvis with bulky mesorectal tissue in a man can be the cause of worse results of TME.

The quality of mesorectal excision and PCRM are related yet independent prognostic factors. The relationship between $\mathrm{PCRM}+$ and the completeness of the mesorectal excision has been documented by Nagtegaal. In $44 \%$ of patients with pCRM+ a torn mesorectum was observed, while patients with an intact mesorectum exhibited pCRM positivity in $11 \%$ of cases [13].

The study COLOR II, which prospectively compared the laparoscopic and open technique for rectal resection, showed no statistically significant difference in the frequency of pCRM+ between these two groups (93\% of laparoscopically operated patients had negative pCRM vs. $91 \%$ of patients in the open surgery group). Conversely, a difference was observed in the positivity of the PCRM in carcinoma of the lower rectum. A significantly lower number was found in the laparoscopic than in the open approach (the difference was 12.4\%). The difference in PCRM positivity was even more pronounced in the case of abdominoperineal amputation. A positive PCRM was found in $25 \%$ of open procedures, but only in $8 \%$ during laparoscopic procedures [17].

Similar results have also been obtained by the prospective multicenter study COREAN, dealing with the results of the treatment of rectal cancer within $9 \mathrm{~cm}$. Positive pCRM was exhibited in $2.9 \%$ of patients in the laparoscopic resection group, and in the open technique group the figure was 4.1\%. Even from other studies comparing the influence of the chosen surgical techniques to PCRM it is obvious that the results in this category are identical $[14,16,19,20]$.

In a study by Laurent, which included more than $80 \%$ of patients with lesions of the middle and lower rectum, there were no differences in the number 
of positive CRM for both techniques (7\% vs. $6 \%$ ), and thus no differences in local recurrence in both groups ( $3.9 \%$ vs. $5.5 \%$ ). These results were probably achieved due to the high number of R0 resections (92\% vs. 95\%) [21]. However, the results may be affected by including in the overall group patients $(12 \%)$ with tumors of the upper rectum. The difference we found between ypCRM+ after laparoscopic and conventional resection of the rectum for cancer within $10 \mathrm{~cm}(12.5 \%$ and $27.8 \%$, respectively) was not statistically significant. There is, however, the assumption that increasing the number of patients in the study resulted in a statistically significant difference in favor of laparoscopic resection. Partial selection of the patients who are candidates for laparoscopic resection (patients with a positive response in tumor size on preoperative treatment, no tumor infiltration of surrounding organs and patients in whom bulky tumors were not found) probably affects the outcome of this study.

Achieving complete mesorectal excision is very important in the case of obtaining a negative circumferential resection margin with all consequences of treatment for the patient [22].

Another factor to consider is the impact of positive lymph nodes and radial spread of tumor through the mesorectal tissue on positive pCRM. Patients with $\mathrm{pN}+$ and in whom positive CRM was observed had a damaged mesorectum in $44 \%$ of cases. This is compared to patients with mesorectal excision quality of the third degree and $\mathrm{pN}-$-, where a positive circumferential margin was found in $24 \%$ of cases [13].

In the COREAN study positive CRM after conventional abdominoperineal amputation was described in $8.3 \%$ and $5.3 \%$ for the laparoscopic procedure [7]. In our study we observed positive CRM in the laparoscopically assisted APR in 33.3\%, and in the open procedure in $47.6 \%$. Such a high rate of non-radical performance can be attributed to the relatively small group of patients with amputation.

A higher frequency of positive pCRM has been found in patients with abdominoperineal amputation $(10.2 \%$ to $13.9 \%)$ compared to low anterior resection (3.6\% to $8.7 \%)[23,24]$.

Positive CRM contributes to the increased incidence of local recurrence $(36.5 \%$ vs. $22.3 \%)$ and a shorter overall survival rate ( $52.3 \%$ vs. $65.8 \%$ ) [22, 24]. In our group similar results were confirmed and led to a change in surgical technique for AP amputation.
The distance of the tumor from the anal verge is closely related to the quality of the removed mesorectum. Low anterior resections for tumors of the distal rectum achieve complete excision only in $39 \%$ of cases, compared with $67 \%$ in cases where tumors are located at distances more than $10 \mathrm{~cm}$ from the anus. Similar results were observed in amputation procedures. The APR group had a complete mesorectum in only $34 \%$ of patients. Compare that with low anterior resections, where complete excision was achieved in $73 \%$ of patients. No difference was observed between the genders and age of the patients [13].

Problems during dissection in the perineal phase of the Miles operation can be tumor infiltration of the levator muscle, which can lead to nonradical surgery with $\mathrm{pCRM}$. Specimen perforation during resection is in even more aboral localisation due to poor orientation of the surgeon in the case of a bulky tumor with a close relationship to nearby organs vagina, prostate, urethra or urinary bladder.

Subsequently, the result is low quality mesorectal excision together with more positive CRM. The solution of this problem may be cylindrical excision of the rectum. Laurent compared the long-term oncological outcome between laparoscopic and conventional resection of the rectum and found that, over a 5 -year period, the incidence of local recurrence was not significantly different (3.9\% vs. $5.5 \%$ ). Furthermore, the long-term disease-free survival results are comparable for the two methods [21, 25].

\section{Conclusions}

Our study showed no differences between laparoscopic and open access with regard to oncological outcomes in rectal resections. Evaluating both pCRM and the quality of excision confirmed the legitimacy of the laparoscopic approach. To confirm these results, further studies on a larger cohort of patients is necessary.

\section{Acknowledgments}

This article was supported by MH CZ - DRO (UHHK, 00179906).

\section{References}

1. Quirke P, Steele R, Monson J, et al. Effect of the plane of surgery achieved on local recurrence in patients with operable rectal cancer: a prospective study using data from the MRC CRO7 and NCIC-CTG CO 16randomised clinical trial. Lancet 2009; 373: $821-8$. 
2. Nagtegaal ID, Quirke P. What is the role for circumferential margin in the modern treatment of rectal cancer. Br J Surg 2009; 96: 982-9.

3. Ströhlein MA, Grützner KU, Jauch KW, et al. Comparison of laparoscopic vs. open access surgery in patients with rectal cancer: a prospective analysis. Dis Colon Rectum 2008; 51: 385-91.

4. Leonard D, Penninckx F, Fieuws S, et al. Factors predicting the quality of total mesorectal excision for rectal cancer. Ann Surg 2010; 252: 982-8.

5. Kellokumpu IH, Kairaluoma MI, Nuorva KP, et al. Short- and long-term outcome following laparoscopic versus open resection for carcinoma of the rectum in the multimodal setting. Dis Colon Rectum 2012; 55: 854-63.

6. Fukunaga Y, Higashino M, Tanimura S, et al. Laparoscopic rectal surgery for middle and lower rectal cancer. Surg Endosc 2010; 24: 449-57.

7. Kang SB, Park JW, Jeong SY, et al. Open versus laparoscopic surgery for mid or low rectal cancer after neoadjuvant chemoradiotherapy (COREAN trial): short-term outcomes of an open-label randomised controlled trial. Lancet Oncol 2010; 11: 637-45.

8. Lee SD, Park SC, Park JW, et al. Laparoscopic versus open surgery for stage I rectal cancer: long-term oncologic outcomes. World J Surg 2013; 37: 646-51.

9. D’Annibale A, Pernazza G, Monsellato I, et al. Total mesorectal excision: a comparison of oncological and functional outcomes between robotic and laparoscopic surgery for rectal cancer. Surg Endosc 2013; 27: 1887-95.

10. Örhalmi J, Klos D, Jackanin S, et al. Intersphincteric resection of the rectum. Rozhl Chir 2012; 91: 101-4.

11. Skrovina, M, Duda, M, Srovnal, J, et al. Evaluation of laparoscopic resection of colorectal carcinoma from the viewpoint of molecular biology. Videosurgery Miniinv 2012; 7: 19-26.

12. Gonzalez QH, Rodriguez-Zentner HA, Moreno-Berber JM, et al. Laparoscopic versus open total mesorectal excision: a nonrandomized comparative prospective trial in a tertiary center in Mexico City. Am Surg 2009; 75: 33-8.

13. Nagtegaal ID, van de Velde CJ, van der Worp E, et al. Macroscopic evaluation of rectal cancer resection specimen: clinical significance of the pathologist in quality control. J Clin Oncol 2002; 20: 1729-34.

14. Hotta T, Yamaue H. Laparoscopic surgery for rectal cancer: review of published literature 2000-2009. Surg Today 2011; 41: 1583-91.

15. Herzog T, Belyaev O, Chromik AM, et al. TME quality in rectal cancer surgery. Eur J Med Res 2010; 15: 292-6.

16. Krane MK, Fichera A. Laparoscopic rectal cancer surgery: where do we stand? World J Gastroenterol 2012; 18: 6747-55.

17. van der Pas MHGM, Haglind E, Vuesta MA, et al. Laparoscopic versus open surgery for rectal cancer (COLOR II): short-term outcomes of a randomised, phase 3 trial. Lancet Oncol 2013; 14: 210-8.

18. Baik SH, Kim NK, Lee KY, et al. Factors influencing pathologic results after total mesorectal excision for rectal cancer: analysis of consecutive 100 cases. Ann Surg Oncol 2008; 15: 721-8.

19. Bosch SL, Nagtegaal ID. The importance of the pathologist's role in assessment of the quality of the mesorectum. Curr Colorectal Cancer Rep 2012; 8: 90-8.
20. Huang MJ, Liang JL, Wang H, et al. Laparoscopic-assisted versus open surgery for rectal cancer: a meta-analysis of randomised controlled trials on oncologic adequacy of resection and longterm oncologic outcomes. Int J Colorectal Dis 2011; 26: 415-21.

21. Laurent C, Leblanc F, Wutrich P, et al. Laparoscopic versus open surgery for rectal cancer: long-term oncologic results. Ann Surg 2009; 250: 54-61.

22. Schneider PM, Vallbohmer D, Ploenes $Y$, et al. Evaluation of quality indicators following implementation of total mesorectal excision in primarily resected rectal cancer changed future management. Int J Colorectal Dis 2011; 26: 903-9.

23. Kim JC, Yu CS, Lim SB, et al. Abdominoperineal resection and low anterior resection: comparison of long-term oncologic outcome in matched patients with lower rectal cancer. Int I Colorectal Dis 2013; 28: 493-501.

24. Trakarnsanga A, Gonen M, Shia J, et al. What is the significance of the circumferential margin in locally advanced rectal cancer after neoadjuvant chemoradiotherapy? Ann Surg Oncol 2013; 20: $1179-84$

25. Asoglu O, Balik E, Kunduz E, et al. Laparoscopic surgery for rectal cancer: outcomes in 513 patients. World J Surg 2013; 37: 883-92.

Received: 19.01.2014, accepted: 14.09.2014. 